\title{
INDUCTION HEATING OF VERY THIN METAL PLATES MODELLED BY ELECTRIC VECTOR POTENTIAL
}

A novel way of modelling of induction heating of very thin nonferromagnetic plates exposed by periodically varying magnetic flux is presented. The currents induced in the plate are described by means of the T-electric vector potential. The mathematical model is solved numerically, by a code developed and written by the authors. The methodology is illustrated with an example whose results are discussed.

\section{Introduction}

Induction heating is a process that nowadays belongs to widely spread and well elaborated technologies of heat treatment of metal bodies from both theoretical and practical viewpoints. The process is based on the induction of electric currents (and consequent production of heat) in the body, usually as a consequence of one of the two following mechanisms - exposition of the body by a time variable magnetic field (the most frequent case) or movement of the body in a static magnetic field.

The theory of induction heating is well known. The principal mathematical model of this process consists of two partial differential equations describing the distribution of magnetic and temperature fields in the system. Magnetic field is usually formulated in terms of magnetic vector potential $\boldsymbol{A}$ [1-3], while temperature field is described by the heat transfer equation $[4,5]$.

Nevertheless, from time to time we must face problems where this classical way of solution fails. Such problems are typically characterized by geometrical incommensurability. This means that one dimension of the investigated system is much smaller than the other dimensions, but it is also important and cannot be neglected. We can mention, for instance:

- nonferromagnetic plate 1 of very small thickness $\delta$ locally exposed by a time varying irrotational magnetic field $\boldsymbol{B}_{\text {ext }}$ generated in magnetic circuit $\mathbf{2}$ by field coil $\mathbf{3}$ and concentrated by appropriate magnetic focusators $\mathbf{4 . 1}$ and $\mathbf{4 . 2}$, see Fig. 1,

- electrically conductive band $\mathbf{1}$ of very small thickness $\delta$ moving at a velocity $\boldsymbol{w}$ between two systems of direct current-carrying field coils or appropriately oriented permanent magnets $\mathbf{2 . 1}$ and 2.2 (see Fig. 2).
Handling such a problem as a geometrically 3D task (dependent on quantities $x, y, z, t$ ), formulating it in the classical manner (in terms of magnetic vector potential $\boldsymbol{A}$ ) and solving it by the finite element method is often unreal. This is because the thickness $\delta$ of the plate (or band) is negligible with respect to its remaining dimensions, which represents the fundamental complication for building the finite-element mesh. Moreover, the definition area of the task is three-dimensional and often large, which leads to a long computation time.

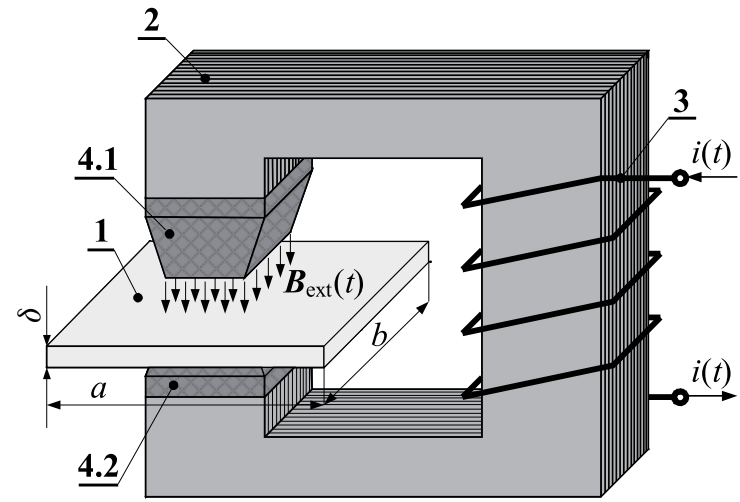

Fig. 1 Induction heating of a very thin plate $(\delta<<a, b)$ : 1-locally heated thin nonferromagnetic plate,

2-laminated magnetic circuit, 3-field coil,

4.1, 4.2-ferromagnetic focusators of magnetic field

\footnotetext{
* Ivo Dolezel ${ }^{1}$, Bohus Ulrych ${ }^{2}$

${ }^{1}$ Department of Electrical Power Engineering, Faculty of Electrical Engineering, Czech Technical University, Czech Republic,

E-mail: dolezel@fel.cvut.cz

${ }^{2}$ Department of Theory of Electrical Engineering, Faculty of Electrical Engineering, University of West Bohemia, Czech Republic
} 


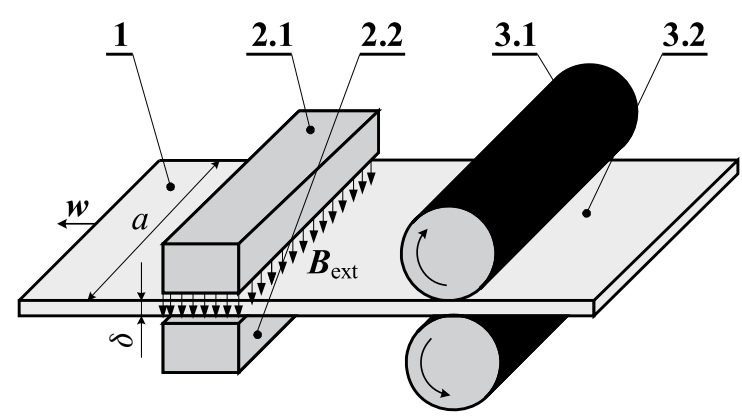

Fig. 2 Induction heating of a very thin electrically conductive band $\delta<<$ a moving at a velocity $\boldsymbol{w}$ in time invariable magnetic field generated by a system of permanent magnets: 1-moving band, 2.1, 2.2-

systems of permanent magnets, 3.1, 3.2-front roll stand

On the other hand, considering the problem as a $2 \mathrm{D}$ task described by the magnetic vector potential $\boldsymbol{A}$ (thickness $\delta$ being neglected) is also counterproductive because it is not possible to numerically approximate the boundary conditions for this quantity along the plate.

In similar cases it is often advantageous to use for modeling of the electromagnetic field the electric vector potential $T$, as is shown in this paper.

\section{Formulation of the Problem}

Consider a very thin nonferromagnetic and electrically conductive circular plate of thickness $\delta \rightarrow 0$ and electrical conductivity $\gamma_{\mathrm{el}}$, whose surface is denoted as $\Omega_{1}$ and its boundary $\Gamma$ (see Fig. 3 ). The plate is locally (in a subdomain $\Omega_{2} \subset \Omega_{1}$ ) exposed by a time variable external irrotational magnetic field $\boldsymbol{B}_{\text {ext }}(t)$.

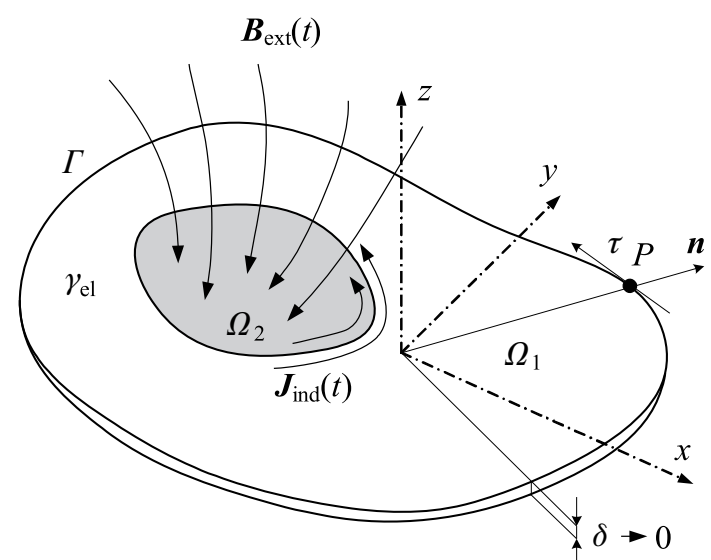

Fig. 3 General arrangement of a very thin $(\delta \rightarrow 0)$ nonferromagnetic plate exposed by local time variable magnetic field

Denoting the electric field strength in the plate $\boldsymbol{E}$, the corresponding eddy current density induced in it is

$$
J_{\text {ind }}=\gamma_{\text {el }} E
$$

and the corresponding volumetric Joule losses are

$$
w_{\mathrm{J} \text {,ind }}=\frac{1}{\gamma_{\mathrm{el}}}\left|J_{\mathrm{ind}}\right|^{2} .
$$

The aim of the paper is to determine the distribution of $\boldsymbol{J}_{\text {ind }}$ and $w_{\mathrm{J} \text {,ind }}$ in the plate, whose knowledge is the crucial condition for finding the time evolution of its temperature.

If the problem were solved in the classical way using magnetic vector potential $\boldsymbol{A}$, the quantity $\boldsymbol{B}_{\text {ext }}(t)$ would be a boundary condition over the locally exposed surface $\Omega_{2}$ of the heated plate. But in this case the plate would have to be considered $3 \mathrm{D}$ and when its thickness $\delta$ would be small, meshing of the plate could cause crucial difficulties.

The method described in the paper uses the electric vector potential $\boldsymbol{T}$. As we will see in the next section, this allows the external irrotational magnetic magnetic flux density $\boldsymbol{B}_{\text {ext }}(t)$ becoming the right-hand part of the partial differential equation describing the distribution of $\boldsymbol{T}$. The numerical discretization is now only carried out in the $2 \mathrm{D}$ area $\Omega_{2}(x, y)$, no matter how small is the thickness $\delta$. The only disadvantage of this approach is that the density of eddy currents along the thickness of the plate is uniform. But with respect to very small value of $\delta$ this error plays no significant role.

\section{Continuous Mathematical Model}

Introduce the electric vector potential $T$ by

$$
E=-\operatorname{curl} T .
$$

Now the second Maxwell equation describing the electric field in the plate reads

$$
\operatorname{curl} E=-\operatorname{curl}(\operatorname{curl} T)=-\frac{\partial B}{\partial t},
$$

where $B$ is the total flux density in the plate. This consists of two parts: the mentioned external magnetic flux density $B_{\text {ext }}$ from the external source and magnetic flux density $B_{\text {ind }}$ produced by the induced eddy currents $J_{\text {ind }}(1)$. Now, we can transform (4) into the form

$$
\operatorname{curl}(\operatorname{curl} T)=\frac{\partial B_{\text {ext }}}{\partial t}+\frac{\partial B_{\text {ind }}}{\partial t} .
$$

Using the first Maxwell equation as the relation between $J_{\text {ind }}$ and $H_{\text {ind }}$ (the displacement currents being neglected)

$$
J_{\text {ind }}=\operatorname{curl} H_{\text {ind }}
$$

we can write

$$
J_{\text {ind }}=\gamma_{\mathrm{el}} E=-\gamma_{\mathrm{el}} \operatorname{curl} T=\frac{1}{\mu_{0}} \operatorname{curl} B_{\text {ind }},
$$


and hence

$$
T=-\frac{1}{\gamma_{\mathrm{el}} \mu_{0}} B_{\text {ind }}-\operatorname{grad} \psi,
$$

where $\psi$ is an arbitrary scalar function. But in our case $T$ represents the electric vector potential only produced by the induced magnetic flux density $B_{\text {ind }}$. That is why we can put $\psi=0$ and

$$
T=-\frac{B_{\text {ind }}}{\gamma_{\mathrm{el}} \mu_{0}} \Rightarrow B_{\text {ind }}=-\gamma_{\mathrm{el}} \mu_{0} T .
$$

After inserting (9) into (5) we obtain the fundamental equation for the electric vector potential in the form

$$
\operatorname{curl}(\operatorname{curl} T)+\gamma_{\mathrm{el}} \mu_{0} \frac{\partial T}{\partial t}=\frac{\partial B_{\mathrm{ext}}}{\partial t} .
$$

The initial and boundary conditions for potential $T$ can be derived from the physical aspects of the problem.

The initial condition follows from the fact that before the plate is exposed by external magnetic field, $B_{\text {ext }}\left(\Omega_{2}, t=0\right)=0$, so that $E\left(\Omega_{1}, t=0\right)=0$, and, hence, $\operatorname{curl} T=0$. This results in $T=$ $\operatorname{grad} \Phi$, where $\Phi$ is any scalar function. Without any loss of generality we can put $\Phi=0$, so that $T\left(\Omega_{1}, t=0\right)=0$.

The boundary condition follows from the fact that the currents induced in the plate in the direction of its any outward normal to the plate vanish $\left(J_{n, \text { ind }}(\Gamma, t)=0\right.$. This gives $-\gamma_{\mathrm{el}} \cdot \partial T(\Gamma, t) / \partial \tau=$ $=0 \Rightarrow T(\Gamma, t)=C$ (symbol $\tau$ denoting the tangent, see Fig. 3 ), where $C$ is a constant vector. In order to preserve the consistency with the above initial condition $T\left(\Omega_{1}, t=0\right)=0$, we immediately obtain $C=0$.

From the above formulas we can see two principal advantages of introducing the electric $T$ potential for such kinds of problems:

- Potential $T$ is only defined in the electrically conductive domain, in our case only in the plate. On the other hand, the magnetic vector potential $A$ would have to be determined everywhere in the whole system (i.e., inductor, magnetic cores, flux concentrators, ambient air), which would require much larger 3D mesh. The transversal discretisation of the thin plate, moreover, could lead to complications.

- The boundary conditions for the electric vector potential $T$ can be derived very easily. On the other hand, finding the value of the magnetic vector potential $A$ along the boundary $\Gamma$ is impossible and for solution we would have to introduce a sufficiently distant artificial Dirichlet boundary.

- The only drawback of the presented approach is that the currents induced in the disk have the same density along its thickness. But this is not very significant when thickness $\delta$ of the plate is substantially smaller than the depth of penetration.

Solution of (10) provides the distribution of the electric vector potential $T$ and, consequently, distribution of all remaining electric and magnetic quantities in the plate. Distribution of current density in the plate is then given by formula

$$
J_{\text {ind }}=\gamma E=-\gamma_{\text {el }} \operatorname{curl} T
$$

and the corresponding volumetric Joule losses are

$$
w_{\mathrm{J}}=\gamma_{\mathrm{el}}|\operatorname{curl} T|^{2} \text {. }
$$

\section{Analogy with Magnetic Vector Potential for Planar Plates}

In this section we will show an important analogy of equation (10) with the equation for vector potential $A$ in case that the plate under inspection is planar (it lies in the plane $x, y$ in Cartesian coordinates) and vector $B_{\text {ext }}$ is perpendicular to it (it is parallel with the $z$-axis.

For this specific case equation (10) can be written in the form

$$
\frac{\partial^{2} T_{z}}{\partial x^{2}}+\frac{\partial^{2} T_{z}}{\partial y^{2}}-\gamma_{\mathrm{el}} \mu_{0} \frac{\partial T_{z}}{\partial t}=-\frac{\partial B_{z, \mathrm{ext}}}{\partial t},
$$

because now both vectors $T$ and $B_{\text {ext }}$ have only the -components $T_{z}$ and $B_{z, \mathrm{ext}}$.

Consider another situation. An infinitely long massive nonmagnetic cylindrical body $V_{1}$ (with the axis identical with axis $z$ ) of electric conductivity $\gamma_{\mathrm{el}}$ and cross section $\Omega_{1}(x, y)$ carries timevariable electric current $i(t)$ of density $\gamma_{z, \text { ext }}(x, y, t)$ that passes in the $z$-direction through body $V_{2}$ of cross section $\Omega_{2}$ (see Fig. 3). The region $\Omega_{2}$ is insulated from $\Omega_{2}-\Omega_{2}$ by a perfect, infinitely thin insulating layer.

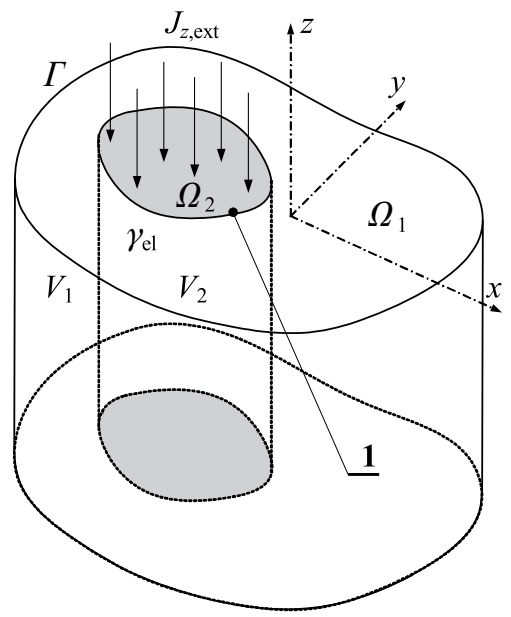

Fig. 4 Infinitely long nonmagnetic cylindrical body carrying timevarying current: 1-infinitely thin perfectly insulating interface

Now, the time variable magnetic field in the whole body produced by current $i(t)$ can be described by the well-known partial differential equation of parabolic type for magnetic vector potential $A$ (that also exhibits only one nonzero component $A_{z}$ in the $z$-direction) in the form

$$
\frac{\partial^{2} A_{z}}{\partial x^{2}}+\frac{\partial^{2} A_{z}}{\partial y^{2}}-\gamma_{\mathrm{el}} \mu_{0} \frac{\partial A_{z}}{\partial t}=-\mu_{0} J_{z, \mathrm{ext}}
$$


It is obvious that equations (13) and (14) are quite analogous. The same holds for the derived quantities: in case of the electric vector potential $T$ we have

$$
J_{x, \text { ind }}=\gamma_{\mathrm{el}} \frac{\partial T_{z}}{\partial y}, J_{y, \text { ind }}=-\gamma_{\mathrm{el}} \frac{\partial T_{z}}{\partial x}
$$

while in case of the electric vector potential $A$ we analogously obtain

$$
B_{x}=\frac{\partial A_{z}}{\partial y}, B_{y}=\frac{\partial A_{z}}{\partial x} .
$$

This analogy results in the fact that the indicated linear problem described in terms of the electric vector potential $T_{z}$ can easily be solved by any software working with the magnetic vector potential $A_{z}$ (while the codes working with the magnetic vector potential $A$ abound, practically no professional code - except for user's programs - works with the electric vector potential $T$ ). We can proceed in the following way:

- First we model the arrangement in Fig. 4. Then we substitute for $J_{z, \text { ext }}$ the value $\frac{1}{\mu_{0}} \frac{\partial B_{z, \text { ext }}}{\partial t}$ and put $A_{z}\left(\Gamma_{1}, t\right)=0$.

- After computing the distribution of $A_{z} \approx T_{z}$ using (14), we calculate the distribution of $B_{x}, B_{y}(16)$ and multiplying them by $\gamma_{\mathrm{el}}$ we immediately obtain the corresponding distribution of $J_{x, \text { ind }}$ and $J_{y, \text { ind }}$.

\section{Illustrative Example}

Consider a thin circular aluminum plate (Fig. 5) of dimensions $\delta=0.001 \mathrm{~m}, r_{\max }=0.1 \mathrm{~m}$ and electrical conductivity $\gamma_{\mathrm{el}}=3.4 \times 10^{7} \mathrm{~S} / \mathrm{m}$. The plate is exposed by an external magnetic field $B_{z, \text { ext }}(t)$ in a concentric circle of radius $r_{\min }=0.02 \mathrm{~m}$.

The time evolution of magnetic flux density $B_{z, \text { ext }}(t)$ is described by periodic saw-like oscillations whose parameters follow from Fig. 6.

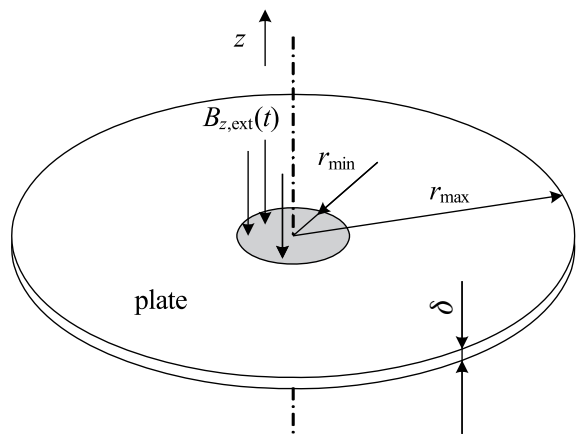

Fig. 5 Thin aluminum circular plate exposed by time-variable magnetic field

The aim of the solution is to determine the spatial and temporal distribution (module of the corresponding vector) of the induced

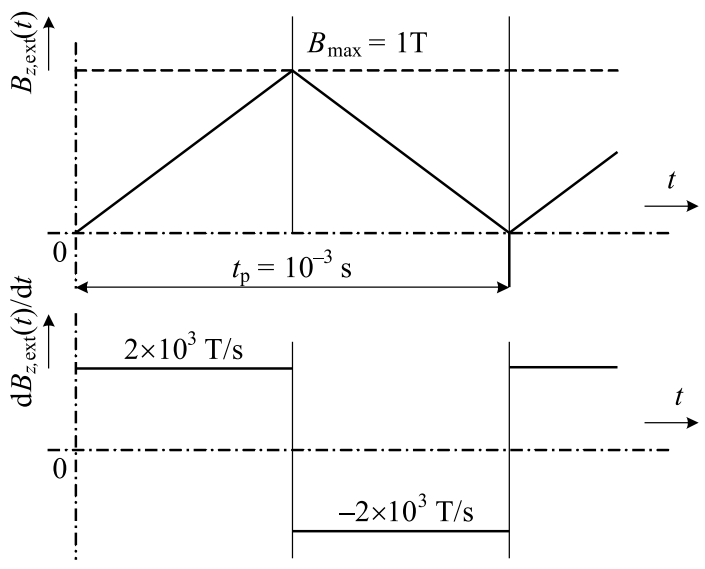

Fig. 6 Time evolution of magnetic flux density $B_{z \text { ext }}(t)$ and its time derivative $\left(f=1000 \mathrm{~Hz}, t_{\mathrm{p}}=0.001 \mathrm{~s}, B_{\max }=1 \mathrm{~T}\right)$

current density $J_{\varphi, \text { ind }}(r, t)(\varphi$ denoting the circumferential direction) and volumetric Joule losses $w_{\mathrm{J}}(r, t)$.

All the results presented in the following figures correspond to the situation in the $10^{\text {th }}$ period, which practically means the steady state (with one exception that will be discussed later).

The orientation of the induced current density $J_{\text {ind }}(r, t)=$ $=\varphi_{0} J_{p, \text { ind }}(r, t)$ in the period is obvious from Figs. 7a, 7b. It is connected with the change of sign of the time derivative $\mathrm{d} B_{z, \text { ext }}(t) / \mathrm{d} t$, see Fig. 6.

The time evolution of module $J_{p \text {,ind }}(r, t)$ along different radii of the plate is depicted in Fig. 8. The highest values are on the circle of $r=r_{\min }=0.02 \mathrm{~m}$, i.e., along the boundary of the domain exposed by the external magnetic field $B_{z, \text { ext }}(t)$. But not too far from this boundary, for example along the radii $r=0.015 \mathrm{~m}$ or $r=0.025 \mathrm{~m}$, its values are significantly lower.

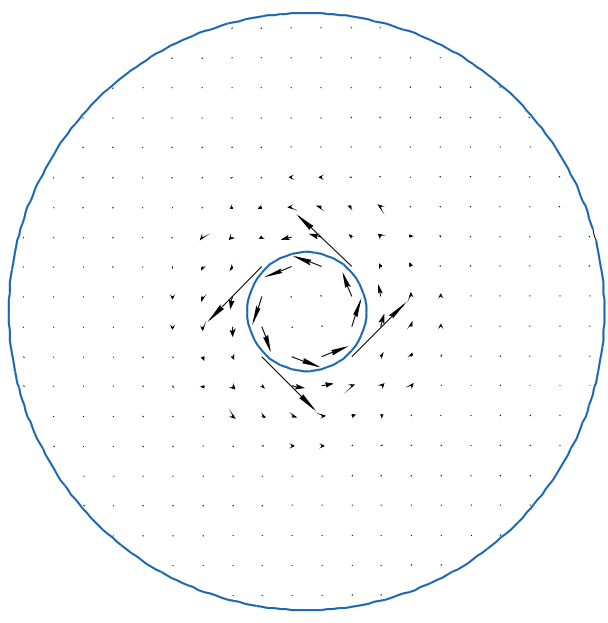

Fig. 7a Distribution of the induced current density $J_{\text {ind }}(r, t)$ in the plate in the first half of the period 


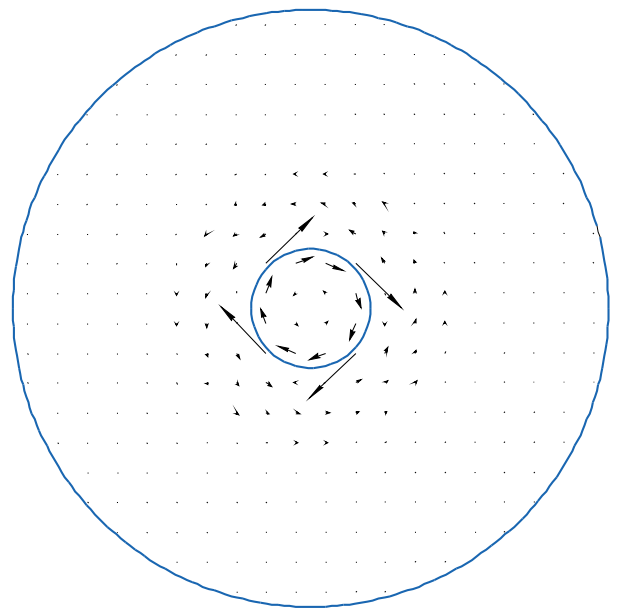

Fig. $7 b$ Distribution of the induced current density $J_{\text {ind }}(r, t)$ in the plate in the second half of the period

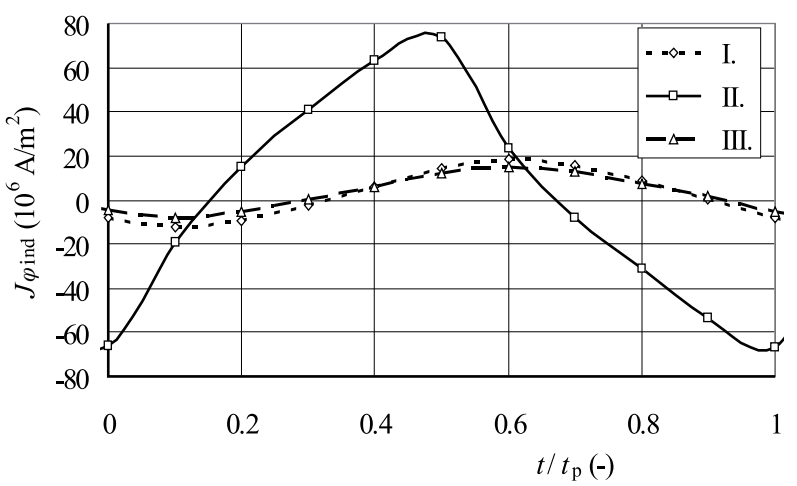

Fig. 8 Time evolution of $J_{p \text {,ind }}(r, t)$ for three various radii of the plate within the $10^{\text {th }}$ period ( being the relative time in this period)

I. $-r=0.015 \mathrm{~m}$, II. $-r=0.02 \mathrm{~m}$, III. $-r=0.025 \mathrm{~m}$

The distribution of module $J_{\varphi \text {,ind }}(r, t)$ of vector $J_{\text {ind }}$ along the radius $r$ of the plate at different time levels of the $10^{\text {th }}$ period, depicted in Fig. 9, also confirms the above conclusions. The figure shows that the highest values of the induced current density occur just on the radius $r=r_{\min }=0.02 \mathrm{~m}$, i.e., on the external boundary of the area exposed by the magnetic flux density $B_{z, \text { ext }}(t)$. These values vary with time, similarly as the mentioned magnetic flux density.

The distribution of the volumetric Joule losses $w_{J, \text { ind }}(r, t)$ along different radii of the plate within the $10^{\text {th }}$ period (depicted in Fig. 10) also confirms the above statements. The highest losses are also produced on radius $r=r_{\text {min }}=0.02$. On the other hand, observable is there certain disproportion between the value $w_{\mathrm{J}, \text { ind }}(r=$ $\left.=0.02, t / t_{\mathrm{p}}=0.5\right)$ and other value $w_{\mathrm{J}, \text { ind }}\left(r=0.02, t / t_{\mathrm{p}}=1\right)$, where $t / t_{\mathrm{p}}$ is relative time in the period of length $t_{\mathrm{p}}$. This is probably connected with the asymmetry of the distribution of $J_{\varphi \text {,ind }}(r, t)$ (see Fig. 8). It is obvious that the $10^{\text {th }}$ period is not yet the ideal steady state.

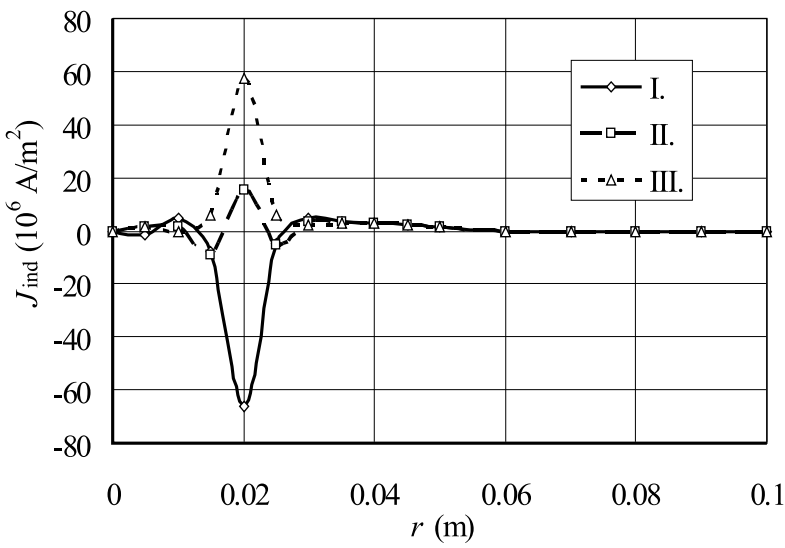

Fig. 9 Time evolution of $J_{\varphi \text {,ind }}(r, t)$ for three various radii of the plate within the $10^{\text {th }}$ period

I. $-t / t_{\mathrm{p}}=0$, II. $-t / t_{\mathrm{p}}=0.2$, III. $-t / t_{\mathrm{p}}=0.4$

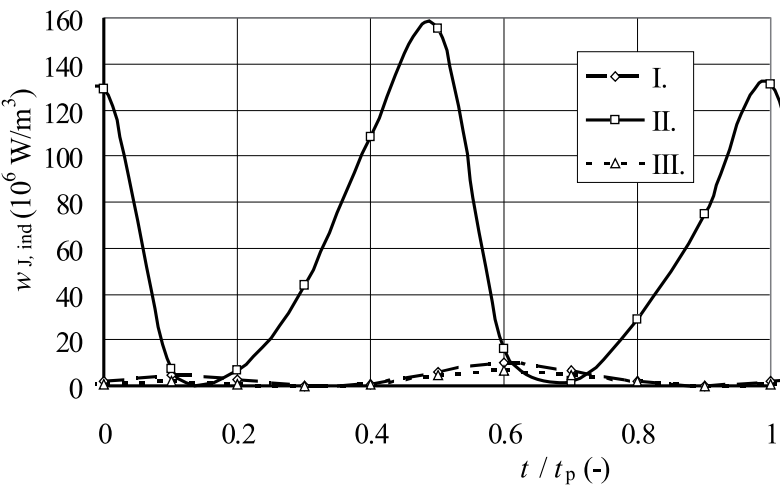

Fig. 10 Time evolution of volumetric Joule losses $w_{\mathrm{J}, \text { ind }}(r, t)$ for three various radii of the plate within the $10^{\text {th }}$ period $\left(t / t_{\mathrm{p}}\right.$ being the relative time in this period)

I. $-r=0.015 m$, II. $-r=0.02 m$, III. $-r=0.025 m$

\section{Conclusion}

Electric vector potential seems to be a powerful tool for modeling induction heating in linear systems characterized by geometrically incommensurable elements, because it allows handling specific $3 \mathrm{D}$ arrangements as $2 \mathrm{D}$ problems. Another advantage consists in the formal similarity of equations for the electric vector potential and magnetic vector potential, which makes possible to use classical FEM codes for numerical computation of such tasks.

Next work in the field will be aimed at the following problems:

- Application of the method in case that the external magnetic field is generally rotational.

- Model of heating taking into account the temperature variations of physical parameters of the system.

- Modeling of the whole problem in the hard-coupled formulation (heating of the plate affects its electrical conductivity) together 


\section{COMMNICOIIONS}

with the thermoelastic changes (leading to small variations of its dimensions) using our own library Hermes [6] with interface Agros based on a fully adaptive higher-order finite element method.

\section{Acknowledgement}

The financial support of the Grant Agency of the Czech Republic (project No. 102/09/1305) and Research Plan MSM 6840770017 is gratefully acknowledged.

\section{References}

[1] ZINN, S., SEMIATIN, S. L.: Elements of Induction Heating: Design, Control and Applications, ASM International, USA, 1988.

[2] RAPOPORT, E., PLESHIVTSEVA, Y.: Optimal Control of Induction Heating Processes. CRC Press, Boca Raton, FL, 2007.

[3] RUDNEV, V. I., LOVELESS, D., COOK, R., BLACK, M.: Handbook of Induction Heating, Marcel Dekker, New York, NY, 2003.

[4] HOLMAN, J. P.: Heat Transfer, McGrawHill, New York, NY. 2001.

[5] KOVACIK, O., ORSANSKY, P.: Partial Differential Equation for Heat Conduction and its Solvability, Communicastions - Scientific Letters of the University of Zilina, 2010, No. 1, pp. 20-22.

[6] hpfem.org. 